\title{
Calculation of Thermodynamic Properties of Liquid Alkali Metals by the First-Principle-Pseudopotential and Weeks-Chandler-Andersen Methods
}

\author{
V. A. Krashaninin, N. E. Dubinin, and Academician N. A. Vatolin \\ Received April 8, 2013
}

DOI: $10.1134 / \mathrm{S} 1028335813080144$

The methods of thermodynamic perturbation theory (TPT) combined with the effective ion-pair interaction considered within the framework of the nearlyfree-electron approximation based on the pseudopotential approach have actively been used for calculating the thermodynamic properties of metal melts for many years [1-8]. In this case, if model pseudopotentials were used for these purposes in combination with various TPT methods (variational [1-6], WeeksChandler-Andersen [9] (WCA) [1, 2, 6, 7], and Barker-Henderson [2]), the first-principle ones were used only with the variational methods $[3,4,8]$.

In this study, together with the WCA method, we used the first-principle pseudo-potential method [10] previously proposed by us, which enables us to consider a very wide circle of properties and gives good quantitative and qualitative results for simple metals and their alloys in the solid state [11]. In this case, both the energy functions and their first and second derivatives agree well with the experimental data. Recently, this pseudo-potential was used for calculating the thermodynamic properties of liquid alkali metals within the framework of the variational method [8].

The Schrödinger pseudo-wave equation for conduction electrons has the form

$$
H_{p s} \phi_{\mathbf{k}}=\varepsilon_{\mathbf{k}} \phi_{\mathbf{k}}=\left(K+W_{\mathbf{k}}\right) \phi_{\mathbf{k}}
$$

where $H_{p s}$ and $\phi_{\mathbf{k}}$ are called the pseudo-Hamiltonian acting on conduction electrons and their pseudo-wave functions, respectively; $\mathbf{k}$ is the conduction-electron state, $W_{\mathbf{k}}$ is the pseudo-potential acting on the electron state $\mathbf{k}$, and $K$ is the kinetic-energy operator. The transformation of the true Schrodinger equation into the pseudo-wave equation is exact, and the eigenvalues $\varepsilon_{\mathbf{k}}$ of Eq. (1) coincide precisely with the eigenval-

Institute of Metallurgy, Ural Branch, Russian Academy of Sciences, Yekaterinburg, 620016 Russia

Ural Federal University, Yekaterinburg, 620002 Russia e-mail:ned67@mail.ru ues $E_{\mathrm{k}}$ of the true Schrodinger equation for conduction electrons. In this case, the pseudo-wave functions $\phi_{\mathbf{k}}$ are related to the true wave functions $\psi_{\mathbf{k}}$ by the formula

$$
\psi_{\mathbf{k}}=\sum_{\mathbf{q}} a_{\mathbf{q}}(\mathbf{k})(1-P)|\mathbf{q}\rangle=(1-P) \varphi_{\mathbf{k}},
$$

where $|\mathbf{q}\rangle$ is the plane wave, $a_{\mathbf{q}}(\mathbf{k})$ are the expansion coefficients, and $P$ is the operator of the projection onto the core-electron states.

The pseudo-potential has the following form:

$$
W_{\mathbf{k}}=V+\sum_{n, t_{n}}\left(\varepsilon_{\mathbf{k}}-E_{n, t_{n}}\right)\left|n, t_{n}\right\rangle\left\langle n, t_{n}\right|,
$$

where $V$ is the true full potential in the system, the subscript $n$ numbers the ion cores in the system, the subscript $t_{n}$ numbers the core states of the $n$th ion core, and $E_{n, t_{n}}$ is the core-state energy. The potential $V$ in the system has the form

$$
V(\mathbf{r})=\sum_{n=1}^{N} v^{0}\left(\mathbf{r}-\mathbf{r}_{n}\right)+V_{\text {scr }}(\mathbf{r}) .
$$

Here, $N$ is the number of ions in the system, $v^{0}\left(\mathbf{r}-\mathbf{r}_{n}\right)$ is the potential of the ion core, which is in the $\mathbf{r}_{n}$ position, and $V_{\mathrm{scr}}(\mathbf{r})$ is the screening potential formed by the conduction electrons and calculated using the true conduction-electron density instead of the pseudo-wave density contrary to previously used approaches [10]:

$$
n(\mathbf{r})=\sum_{\mathbf{k}} \frac{\psi_{\mathbf{k}}^{*}(\mathbf{r}) \psi_{\mathbf{k}}(\mathbf{r})}{A(\mathbf{k})}
$$

where $A(\mathbf{k})$ is the normalizing factor.

The use of the true wave functions of conduction electrons also enables us, contrary to investigations of other authors, to take into account correctly the energy shifts for the core electrons caused by their interaction with the potential formed by the distribution of the conduction-electron charge: 


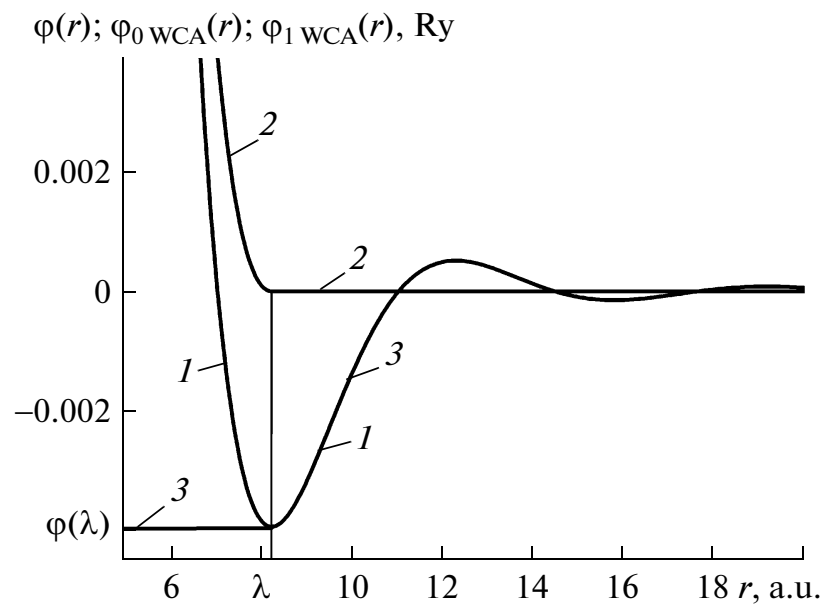

Fig. 1. (1) Effective ion-pair interaction potential, (2) reference-system pair potential, and (3) perturbation obtained for liquid sodium at $\Omega_{0}=278$ a.u.

$$
\Delta \varepsilon_{n, t_{n}}=\Omega_{0} \sum_{\mathbf{q}}|S(\mathbf{q})|^{2} \rho_{n, t_{n}}^{*}(\mathbf{q}) \nu_{\mathrm{scr}}(\mathbf{q}),
$$

where $\Omega_{0}$ is the mean atomic volume, $S(\mathbf{q})$ is the structure factor, $\rho_{n, t_{n}}(\mathbf{q})$ are the Fourier components of the charge-density distribution for the electrons under consideration, and $v_{\text {scr }}(\mathbf{q})$ is the form factor of the screening potential.

In this case, the method of solving the Schrodinger pseudo-wave equation, which is exact for the conduction electrons, remains only an approximation. In the case of metals with the filled $d$ shells, we use the perturbation theory. Further, having calculated the true wave functions, we can also determine all other properties. The approximations of the pseudo-potential approach are no longer used, and the passage from the true Schrodinger equation to the pseudo-wave equation is used only as the method of its solution.

The potential of the effective ion-pair interaction obtained to within the second order of the perturbation theory has the following form in the pseudopotential approach under use:

$$
\varphi(r)=2\left(\frac{z^{*}}{z}-\frac{1}{2}\right) \frac{z^{2}}{r}+\frac{\Omega_{0}}{\pi^{2}} \int_{0}^{\infty} F(q) \frac{\sin (q r)}{q r} q^{2} d q,
$$

where $z$ and $z^{*}$ are the valence and effective valence of a metal, respectively, and $F(q)$ is the characteristic function of the band structure [10].

In the TPT, the pair potential $\varphi(r)$ is written as the sum of the reference-system pair potential $\varphi_{0}(r)$ and the perturbation potential $\varphi_{1}(r)$. The method of their determination depends on the particular TPT method. In the WCA method,

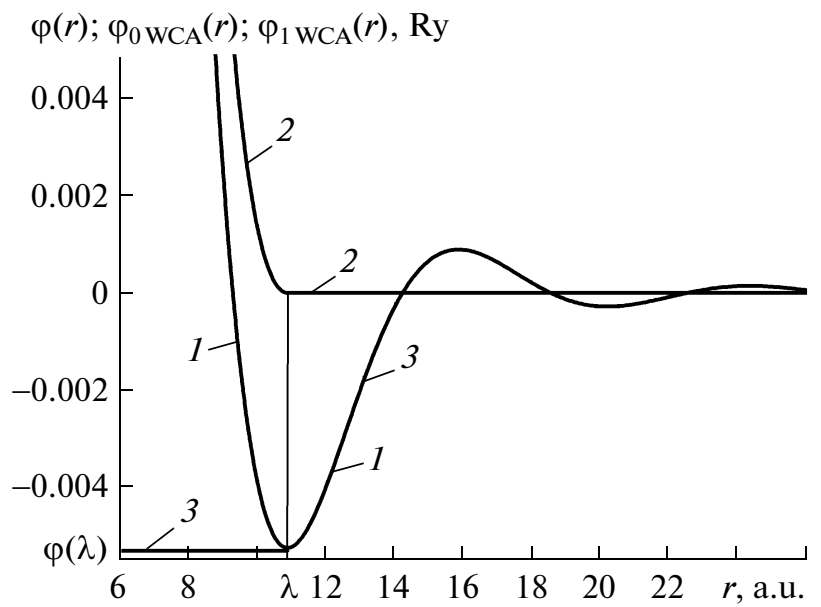

Fig. 2. (1) Effective ion-pair interaction potential, (2) reference-system pair potential, and (3) perturbation obtained for liquid potassium for $\Omega_{0}=529$ a.u.

$$
\begin{gathered}
\varphi_{0 \mathrm{WCA}}(r)= \begin{cases}\varphi(r)-\varphi(\lambda), & r<\lambda, \\
0, & r \geq \lambda,\end{cases} \\
\varphi_{1 \mathrm{WCA}}(r)= \begin{cases}\varphi(\lambda), & r<\lambda, \\
\varphi(r), & r \geq \lambda,\end{cases}
\end{gathered}
$$

where $\lambda$ is the position of the first minimum of $\varphi(r)$. From now on, all values with the subscript 0 are referred to the reference system, and those with the subscript 1 relate to the perturbation.

In Figs. 1 and 2, as an example of division of the pair potential in the WCA method, we present $\varphi(r)$ calculated for $\mathrm{Na}$ and $\mathrm{K}$ from Eq. (7) at the experimental mean atomic volumes near the melting points [12] and the potentials $\varphi_{0 \mathrm{WCA}}(r)$ and $\varphi_{1 \mathrm{WCA}}(r)$ obtained from the mentioned $\varphi(r)$ using Eqs. (8) and (9), respectively.

The Helmholtz energy in the WCA method can be written as follows (from now on, all thermodynamic values are written per atom):

$$
\begin{aligned}
& F_{\mathrm{WCA}} \\
& =F_{\mathrm{var}}+\frac{2 \pi}{\Omega_{0}}\left(\left[\varphi(\lambda)-k_{\mathrm{B}} T\right] \int_{0}^{\infty}\left(g_{0 \mathrm{WCA}}(r)-g_{\mathrm{HS}}(r)\right) r^{2} d r\right. \\
& \left.-\int_{\sigma}^{\lambda} \varphi_{0 \mathrm{WCA}}(r) g_{\mathrm{HS}}(r) r^{2} d r\right),
\end{aligned}
$$

where $F_{\text {var }}$ is the Helmholtz energy in the variational method with the hard-sphere (HS) reference system:

$$
F_{\mathrm{var}}=F_{\mathrm{HS}}+\frac{2 \pi}{\Omega_{0}} \int_{\sigma}^{\infty} \varphi(r) g_{\mathrm{HS}}(r) r^{2} d r
$$


Thermodynamic properties of liquid alkali metals calculated by various methods for optimum values of the HS-model diameters in comparison with experimental data

\begin{tabular}{|c|c|c|c|c|c|c|c|}
\hline Metal & $T, \mathrm{~K}$ & $\begin{array}{c}\Omega_{0},(\text { a.u. })^{3} \\
\text { [12] }\end{array}$ & Method & $F$, Ry & $E$, Ry & $\frac{S}{k_{\mathrm{B}}}$ & $\sigma$, a.u. \\
\hline \multirow[t]{3}{*}{$\mathrm{Li}$} & \multirow[t]{3}{*}{454} & \multirow[t]{3}{*}{151} & WCA & -0.525 & -0.511 & 4.50 & 4.50 \\
\hline & & & variational & -0.514 & -0.506 & 3.27 & 5.36 \\
\hline & & & experiment [15] & -0.525 & -0.509 & 5.65 & - \\
\hline \multirow[t]{3}{*}{$\mathrm{Na}$} & \multirow[t]{3}{*}{371} & \multirow[t]{3}{*}{278} & WCA & -0.468 & -0.452 & 6.61 & 5.64 \\
\hline & & & variational & -0.463 & -0.450 & 5.54 & 6.55 \\
\hline & & & experiment [15] & -0.472 & -0.453 & 7.79 & - \\
\hline \multirow[t]{3}{*}{$\mathrm{K}$} & \multirow[t]{3}{*}{337} & \multirow[t]{3}{*}{529} & WCA & -0.421 & -0.407 & 6.76 & 7.04 \\
\hline & & & variational & -0.396 & -0.382 & 6.51 & 8.21 \\
\hline & & & experiment [15] & -0.403 & -0.384 & 9.06 & - \\
\hline \multirow[t]{3}{*}{$\mathrm{Rb}$} & \multirow[t]{3}{*}{312} & \multirow[t]{3}{*}{651} & WCA & -0.426 & -0.410 & 7.52 & 7.61 \\
\hline & & & variational & -0.382 & -0.367 & 7.80 & 8.77 \\
\hline & & & experiment [15] & -0.385 & -0.364 & 10.25 & - \\
\hline
\end{tabular}

where $k_{\mathrm{B}}$ is the Boltzmann constant, $T$ is the absolute temperature, $g(r)$ is the pair correlation function, and $\sigma$ is the HS diameter,

$$
g_{0 \mathrm{WCA}}(r)=g_{\mathrm{HS}}(r) e^{\beta\left(\varphi_{\mathrm{HS}}(r)-\varphi_{0 \mathrm{WCA}}(r)\right)},
$$

where $\beta=\frac{1}{k_{\mathrm{B}} T}$. If we use the Percus-Yevick (PY) approximation [13] for the HS model,

$$
g_{0 \mathrm{WCA}}(r)= \begin{cases}-c_{\mathrm{HS}-\mathrm{PY}}(r) e^{-\beta \varphi_{0 \mathrm{WCA}}(r)}, & r<\sigma, \\ g_{\mathrm{HS}-\mathrm{PY}}(r) e^{-\beta \varphi_{0 \mathrm{WCA}}(r)}, & \sigma \leq r<\lambda, \\ g_{\mathrm{HS}-\mathrm{PY}}(r), & r \geq \lambda,\end{cases}
$$

where $c(r)$ is the direct correlation function, $c_{\mathrm{HS}-\mathrm{PY}}(r)$ and $g_{\mathrm{HS}-\mathrm{PY}}(r)$ are the expressions in the analytical form obtained in [14].

The contribution $F_{\text {var }}$ is calculated in the reciprocal space. The procedure of calculation of this contribution within the framework of the pseudo-potential approach used is described in $[8,11]$.

A feature of the WCA method is the necessity of using the pair potential also in the direct space (in calculation of the second contribution in the right-hand side of Eq. (10)). In this case, there is no principal difference in precisely which approximation the calculated potential $\varphi(r)$ is used.

The entropy $S$ is calculated from the formula obtained in [7]:

$$
\begin{gathered}
S_{\mathrm{WCA}}=S_{\mathrm{HS}}+\frac{U_{0 \mathrm{WCA}}}{T} \\
+2 \pi \rho k_{\mathrm{B}} \int_{0}^{\infty}\left(g_{0 \mathrm{WCA}}(r)-g_{\mathrm{HS}}(r)\right) r^{2} d r,
\end{gathered}
$$

where

$$
U_{0 \mathrm{WCA}}=2 \pi \rho \int_{0}^{\infty} \varphi_{0 \mathrm{WCA}}(r) g_{0 \mathrm{WCA}}(r) r^{2} d r .
$$

Using $F_{\mathrm{WCA}}$ and $S_{\mathrm{WCA}}$ obtained from Eqs. (10) and (14), respectively, and the relation $F=E-T S$ (where $E$ is the binding energy), we calculated $E_{\mathrm{WCA}}$.

In this study, for determining the value of the HS diameter, we use the method proposed in [2], which consists in minimizing the right-hand side of Eq. (10) with respect to $\sigma$.

The Helmholtz energy, the entropy, and the binding energy for liquid $\mathrm{Li}, \mathrm{Na}, \mathrm{K}$, and $\mathrm{Rb}$ were calculated. In the table, we compared the obtained results with those of calculation by the variational method and with the experimental data [15].

It can be seen that, as a whole, the results of calculations by the WCA method agree well with the experimental data. This agreement for $\mathrm{Li}$ and $\mathrm{Na}$ is much better than that in the case of using the variational method. A certain deterioration of results for the Helmholtz energy and the binding energy for $\mathrm{K}$ and $\mathrm{Rb}$ in comparison with the calculation by the variational method is observed; however, the accuracy of calculation of the entropy remains at the former level in this case. 
Thus, it is possible to reach the conclusion that the combined approach of the first-principle pseudopotential and WCA methods can be used for predicting the thermodynamic properties of liquid metals.

\section{ACKNOWLEDGMENTS}

This work was supported by the Russian Program of Scientific Schools, project no. 1278.2012.3, and by the Russian Foundation for Basic Research, project no. 11-03-01029-a.

\section{REFERENCES}

1. R. Kumaravadivel and R. Evans, J. Phys. C: Solid State Phys. 9, 3877 (1976).

2. A. M. Bratkovsky, V. S. Vaks, and A. V. Trefilov, J. Phys. F: Metal Phys. 13, 2517 (1983).

3. J. A. Moriarty, D. A. Young, and M. Ross, Phys. Rev. B 30, 578 (1984).

4. J. Hafner, The Electronic and Statistical-Mechanical Theory of s, $p$ Bonded Metals and Alloys (Springer, Berlin, 1987).
5. N. E. Dubinin, A. A. Yuryev, and N. A. Vatolin, High Temp. Mater. Processes 14 (4), 285 (1995).

6. N. E. Dubinin, A. A. Yuryev, and N. A. Vatolin, Thermochim. Acta 316, 123 (1998).

7. N. E. Dubinin, A. A. Yuryev, and N. A. Vatolin, Thermochim. Acta 518, 9 (2011).

8. V. A. Krashaninin, A. A. Yur'ev, and E. A. Yur'ev, Rasplavy, No. 4, 24 (2011).

9. J. D. Weeks, D. Chandler, and H. C. Andersen, J. Chem. Phys. 54, 5237 (1971).

10. V. A. Krashaninin, Dokl. Akad. Nauk 332 (5), 595 (1993).

11. V. A. Krashaninin, Rasplavy, No. 4, 3 (1999).

12. V. E. Zinov'ev, Thermal Properties of Metals at High Temperatures (Metallurgiya, Moscow, 1989) [in Russian].

13. J. K. Percus and G. Y. Yevick, Phys. Rev. 110 (1), 1 (1958).

14. M. S. Wertheim, Phys. Rev. Lett. 10, 321 (1963).

15. P. I. Bystrov, D. N. Kagan, G. A. Krechetova, and E. E. Shpil'rain, Liquid-Metal Heat Carriers of Thermal Pipes and Energy Installations (Nauka, Moscow, 1988) [in Russian].

Translated by V. Bukhanov 